\section{CONTRASTS IN MIDWIFERY.}

\section{To the Editor of The LANCET.}

Sir:-I herewith send you the history of a well-marked case of spontaneous evolntion of the foetus, which I have thought it might be useful to contrast with one in which the powers of nature were inadequate to the accomplishment of that curious and interesting process.

My practice has furnished me with a treat variety of important, and some nnusual, cases in midwifery. If the specimens which I now send possess sufficient interest to induce you to give them a place in the pages of The Lancet, I shall feel great pleasure in forwarding others for you considerarion. In the mean time, $\bar{I}$ remain, Sir, yours very respectfully, $\dagger$

\section{J. BEDINGFIELD.}

\section{Medical School, Stowmarket,} April $21,1837$.

Arm and Shoulder Presentation. Spontaneous Evolution.-Mrs. C., aged 37, the mother of several children, has generally had easy and natural labours, except on one occasion, a case of placental presentation, in which, copious bxmorrhage supervening, I was obliged to turn the child.

I was again called to Mrs. C. on the 1 st of July, 1832. On examination $I$ found that the membranes were ruptured, the os uteri dilated to the size of a crown-piece, or rather larger, the child presenting at the superior aperture of the pelvis, but so high up that $I$ could not precisely make out the presenting part. It felt to me, however, like the fore-arm lying across what I thought to be the head. A few pains brought the child lower down, and enabled me to ascertain that the part which 1 had mistaken for the fore-arm was the edge of the scapula; and, for the head, the dorsum of that bone; in short, that it was a shoulder presentation, and that, in all probability, in would be necessary to turn the child. Finding, however, the spains strong, and the shoulder rapidly advancing, and knowing the pelvis to be wall formed, I determined to wait for a short time, and watch the operations of nature. The arm was next expelled, and I now made up my mind to afford manual assistance, and rêtired, for an instant, from the bedside, to prepare for the operation of turning. Upon resuming my seat, I was agreeably surprised to find the nates presenting, which, with the feet and one arm, were speedily protruded. Nothing now remained for me to do but to bring down the remaining arm (the right), and to expedite the expulsion of the head. This was readily accomplished, and a large, fullgrown, dead child was produced. The placenta speedily followed, and the patient had a very favourable getting up. 'The whole process of parturition was cornmenced and completed in somewhat less than two hours. The child, I should imagine, had been dead several days, as it was rather flaccid, and the putrefactive process had . commenced about the scrotum.

Presentation of the Arm, Foot, and Funis. -I was requested to see, in consultation, Mrs. B., the mother of several children, but her labours had always been severe and protrated; and it had been necessary on two occasions to deliver with the forceps.

Upon examination, several inches of the funis were found hanging through the os externum, cold, and destitute of pulsation; a foot and arm in the vagina; and the os tincre fully dilated. The membranes had ruptured, and the liquor amnii had escaped many hours, leaving the uterus firmly contracted round the child. Mrs. B. had been in labour about forty-eight hours, and the pains were still powerful, but, from the mal-position of the child, totally insufficient to effect its expulsion.

Under these circumstances, the presenting foot was first brought down and secured by a fillet; but in consequence of the contracted state of the uterus, the narrowness of the pelvis, and the great size of the child, immense difficulty was experienced in accomplishing its delivery. An hour was occupied in steady, persevering, skilful at. tempts, made by the nost experienced and judicious surgeon in the county (Mr. Beck, of Needham Market), before the child could be brought into the world.

Unfortunately severe uterine and peritoneal inflammation supervened, which, notwithstanding the most active treatment, terminated fatally on, I think, the fourth day after delivery.

Remarks.-These two cases present a striking contrast, and very clearly prove that no precise rules can be laid down for the management of such cases. In the first case the evolution was spontaneous and rapid, and the child was expelled with almost as much facility as in a natural presentation. The children in both cases were large, and still-born. The difficulty of effecting delivery in the latter, was entirely to be attributed to the narrowness of the pelvis, and the contracted state of the uterus. 